\title{
Propuesta de diseño para un centro integral de reciclaje con inclusión de fuentes alternativas de energía
}

Integral recycling center involving

alternative sources of energy

Luis Alejandro Arias B. ${ }^{*}$

Edwin Rivas T.**

Cindy Nayid Vega S. ${ }^{* \star *}$

Centre de recyclage utilisant

des sources d'énergie

alternatives

Centro integral de reciclagem

com inc/usãu de fontes

alternativas de energia

*Ingeniero electromecánico, graduado en la República de Ucrania (antigua Unión Soviética). Mestría en Ciencias de la Ingeniería de la Universidad Politécnica estatal de Kharkov,Ucrania. Maestría en Ciencias de la Información y Las Telecomunicaciones con énfasis en Teleinformática, de la Universidad Distrital Francisco fosé de Caldas.

**Doctorado en Ingeniería Eléctrica, Electrónica y Automática-Universidad Carlos III de Madrid Maestría en Sistemas de Generación de Energía Eléctrica-Universidad del Valle. Mestría en Ingeniería Eléctrica, Electrónica y Automática-Universidad Carlos III de Madrid. Ingeniero Eléctrico-Universidad del Valle. 


\section{RESUMEN}

El presente artículo, plantea una solución integral al problema del manejo de basuras en centros urbanos, realizando el reciclaje de los desechos, creando un Cluster económico y generando energía eléctrica de bajo costo con relación a sistemas de generación y distribución actuales. La solución propuesta atiende requerimientos medioambientales y de salubridad, a través de la reducción y procesamiento de basuras, cuya inadecuada disposición puede llegar a afectar la salud humana. Finalmente, busca contribuir al desarrollo socioeconómico de las regiones. Se muestra el diseño de un centro integral de reciclaje con fuentes alternativas de energía, cuya modelación se basa en la metodología del Lenguaje de Modelación Universal (por sus siglas en inglés, $U M L$ ).

\section{ABSTRACT}

This article describes an integral solution to the problem of trash manipulation in urban centers, recycling trash, creating an economic cluster and generating low-cost-electrical energy in relation with the current systems of generation and distribution. This proposal responds to environmental and health requirements through the reduction and processing of trash, whose wrong disposal affects human health and welfare, finally contributing to socio- economic development of all regions. A design of an integral recycling center using alternative sources of energy is presented, which focuses on the application of a universal modeling language (UML).

\section{RESUMÉÉ}

Cet article présente une solution globale au problème de la gestion des déchets dans les centres urbains grâce au recyclage des résidus, à la création d'un réseau économique autour du du secteur du recyclage et grâce à la production d'énergie électrique à bas coût concurrençant les systèmes actuels de production et de distribution. La solution proposée répond aux exigences environnementales et sanitaires de réduction et de traitement des déchets dont la gestion inadéquate peut affecter négativement la santé publique, et contribue dans le même temps au développement socio - économique des régions. L'article étudie la conception d'un centre de recyclage complet utilisant les sources d'énergie alternative dont la modélisation est basée sur la méthodologie UML (Universal Modeling Language).

\section{RESUMO}

Este artigo propõe uma solução integral para o problema do manejo de lixo em centros urbanos, realizando a reciclagem dos resíduos, criando um cluster econômico e gerando energia elétrica de baixo custo em relação com os sistemas de geração e distribuição atuais. A solução proposta atende requisitos ambientais e de saúde, através da redução e processamento de lixo, cujo descarte inadequado pode afeitar a saúde humana; e finalmente objetiva contribuir ao desenvolvimento socioeconômico das regiões. Mostra-se o desenho de um centro integral de reciclagem com fontes alternativas de energia cujo modelamento está baseado na metodologia UML (Linguagem de modelamento Universal).

\section{Palabras clave}

Reciclaje

Residuos

Cluster económico

$U M L$

Generación distribuida Micro-red.

\section{Keywords}

Recycling

Residuals

Economic cluster

$U M L$

Distributed generation

\section{Mots clefs}

Recyclage

Déchets

Pôle économique

$U M L$

Production décentralisée

Réseau Micro

\section{Palavras-chave}

$$
\begin{aligned}
& \text { Reciclagem } \\
& \text { Resíduos }
\end{aligned}
$$

Cluster econômico

$U M L$

Geração distribuída Micro rede. 


\section{INTRODUCCIÓN}

E ste artículo se deriva de un proyecto de investigación denominado "Diseño de modelo de gestión de redes de distribución con fuentes convencionales y de generación distribuida", Código de registro: Convocatoria No 19 SUI-FUAC "Sistema Unificado de Investigación". Grupo de investigación Energética de la Fundación Universidad Autónoma de Colombia, Bogotá D.C., Colombia.

La mayoría de las ciudades y municipios de nuestro país, han enfrentado por muchos años complejos problemas relacionados con el tratamiento de los desechos, la recolección de basura, la contaminación del medio ambiente y sumado a lo anterior, se tienen aún altos índices de desempleo en el sector urbano y rural cercanos al 9.3\%. Estos problemas se evidencian por la falta de una infraestructura real de desarrollo social y económico (DANE, 2013). Es por ello que se propone una solución integral para el tratamiento de los desechos y que de respuesta a las problemáticas sociales y económicas en materia de empleabilidad y creación de empresa y reducción de los impactos medioambientales. El manejo que debe realizarse de los desechos sólidos y líquidos que se originan en los centros poblacionales, exige especial atención y por ello, se deben tomar medidas que permitan frenar el avanzado proceso de contaminación y proponer soluciones de tratamiento a los desechos que se generan diariamente. En los últimos años, se ha desarrollado una legislación para el manejo de los residuos sólidos, enmarcada dentro de la política nacional para la Gestión de Residuos Sólidos emitida en 1998, actualizada en documentos Conpes y soportada por la Constitución Nacional de Colombia. Las leyes 99 de 1993 y 142 de 1994, establecen objetivos específicos que determinan prioridades en la gestión integral de los residuos que se presentan a continuación.

En primer término, minimizar la cantidad de residuos en zonas urbanas y rurales industriales y residenciales; segundo, incentivar el aprovechamiento racional de los residuos sólidos; y finalmente, mejorar los sistemas de eliminación, tratamiento y disposición final de los residuos sólidos (Aluna, 2011). La legislación estipula también la obligatoriedad de formular por parte de los municipios los Planes de Gestión Integral de Residuos Sólidos (PGIRS), como una herramienta de gestión constituida por una serie de objetivos, metas, programas, proyectos y actividades, definidos por el ente territorial para la prestación del servicio de aseo y el manejo de los residuos sólidos (Ministerio del Medio Ambiente de Colombia, Decreto 1713/2002).

En el presente documento, se propone el diseño de un Centro Integral de Reciclaje de Residuos Sólidos (CIRRS), que se convierta en un eslabón funcional dentro de un Cluster económico en una cadena productiva, y además de ello, que se incluya como un eslabón productivo de la cadena energética. El CIRRS, busca dar solución a problemas sociales, económicos, medioambientales y energéticos de las comunidades, de manera autosostenible. Resulta relevante señalar que el Estado promueve e incentiva las organizaciones del sector industrial e investigativo, para que trabajen sobre el diseño y la puesta en marcha de programas de reciclaje de residuos sólidos (Ministerio del Medio Ambiente de Colombia, Decreto 2695/2000). Se aborda el tema del aprovechamiento y la gestión integral de residuos sólidos, tomando como marco referencial la legislación vigente al respecto. En el aparte de materiales y métodos, se describe la forma como se ha realizado la modelación de la propuesta a través del Lenguaje de Modelación Universal (UML), para luego presentar la estructura general del centro, acompañada de la descripción y estrategias detalladas de las fases del proceso de reciclaje. Posteriormente, se esboza el Cluster económico señalando los productos y las empresas que se derivarían a partir del CIRRS; luego, se aborda el concepto de micro-red energética la cual se formaría a partir del CIRRS con miras a su autoabastecimiento y comercialización de energía; finalmente, se presentan las conclusiones sobre el trabajo de diseño realizado y la viabilidad para la implementación que tiene el proyecto. 


\subsection{Aprovechamiento de residuos sólidos}

Comparado con el vertimiento o encubrimiento y la incineración, el reciclaje de los residuos sólidos resulta un método mucho más amigable con el medio ambiente.

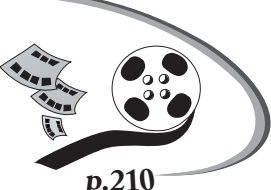

El reciclaje por ejemplo, permite reducir los residuos de elementos como los plásticos, metales, elementos maderables, de vidrio, papel y desechos de construcción, entre otros, permitiendo una sostenible reducción de los requerimientos de producción para nuevo material de los tipos ya mencionados, minimizando el impacto sobre fenómenos como el calentamiento global (Astrup et al., 2009). Buena parte de municipios de nuestro país, utilizan rellenos sanitarios a cielo abierto para la disposición final de desechos, esto debido en gran parte, al desconocimiento de las normas vigentes en materia de manejo de residuos sólidos y a la poca promoción de este tipo de iniciativas por parte de las instituciones gubernamentales encargadas en lo que respecta a las tecnologías de aprovechamiento de recursos sólidos y a la producción limpia en general (Ministerio del Medio Ambiente de Colombia, Ley 99 de 1993, Artículo 5, numeral 32). A contnuación (Figura 1) se observa a manera de ejemplo, un sector del relleno sanitario del Municipio de La Dorada (Caldas); allí, se realiza el vertimiento de la mezcla heterogénea de residuos que posteriormente son cubiertos por películas de PVC y luego se someten a una compactación en terreno por medio de maquinaria pesada. Es de señalar que para el diseño propuesto del CIRRS, se han tomado los datos de desechos de este Municipio durante los meses de abril a julio de 2012, suministrados por funcionarios de la Empresa de Servicios Públicos del Municipio. Los rellenos sanitarios a cielo abierto, son focos de efluentes lixiviados altamente contaminantes, proliferación de malos olores producto de un proceso de descomposición de las basuras no controlado, entre otros fenómenos que sumados a las características climáticas de cada región y a lo heterogéneas que pueden ser las mezclas de residuos (en razón a las épocas del año y a los hábitos de consumo de los agentes poblacionales de la región), constituyen un escenario desolador y con-trario a los objetivos propuestos en la legislación del país en materia de conservación del medioambiente (Ministerio del Medio Ambiente de Colombia, Ley 99 de 1993) (Ministerio del Medio Ambiente de Colombia, Ley de conservación del medioambiente sano título II, Capítulo 3, Artículo 79).

Figura 1. Vertimiento de residuos sólidos a cielo abierto en la zona de La Dorada (Caldas)
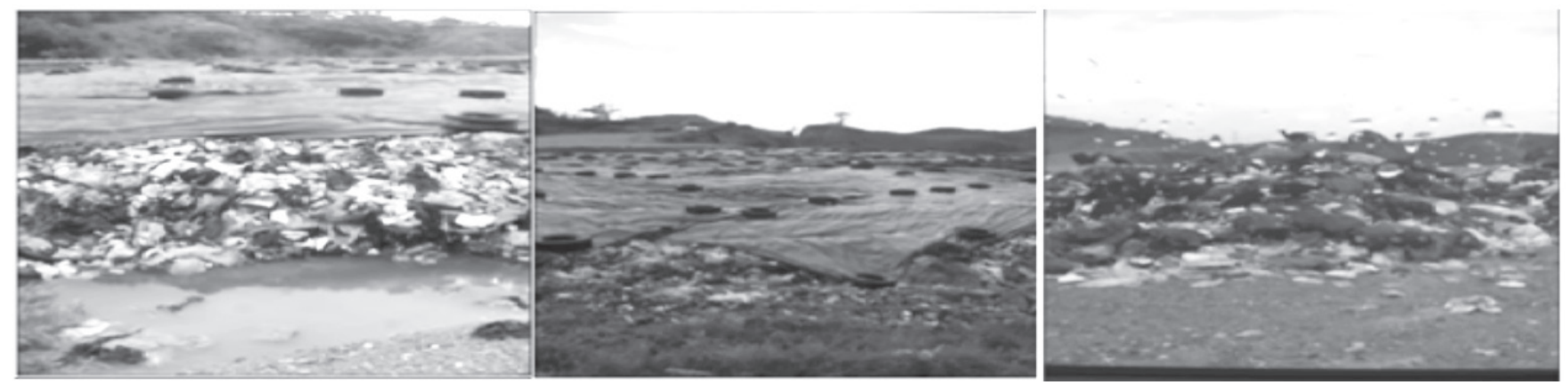

Fuente. Evidencia fotográfica recuperada por los autores, 2014. 


\section{MATERIALES Y MÉTODOS}

$\mathbf{L}$ a presente propuesta, retoma estrategias de reciclaje ya existentes y propone otras etapas de reciclaje como la fabricación de materiales, la obtención de insumos agrícolas de mejor calidad, la producción de biocombustibles y la generación de energía eléctrica en forma alternativa, encaminada inicialmente a suplir las necesidades propias del centro.
A continuación se muestra la modelación del aspecto técnico de la propuesta hecha en UML, ilustrando las fases del proceso de aprovechamiento de residuos sólidos propuesto iniciando con el estudio de los casos de uso, la diagramación de las clases y el diagrama de secuencias del CIRRS (figuras 2,3 y 4).

Figura 2. Diagrama de casos de uso para la estructura del proceso tecnológico global del CIRRS

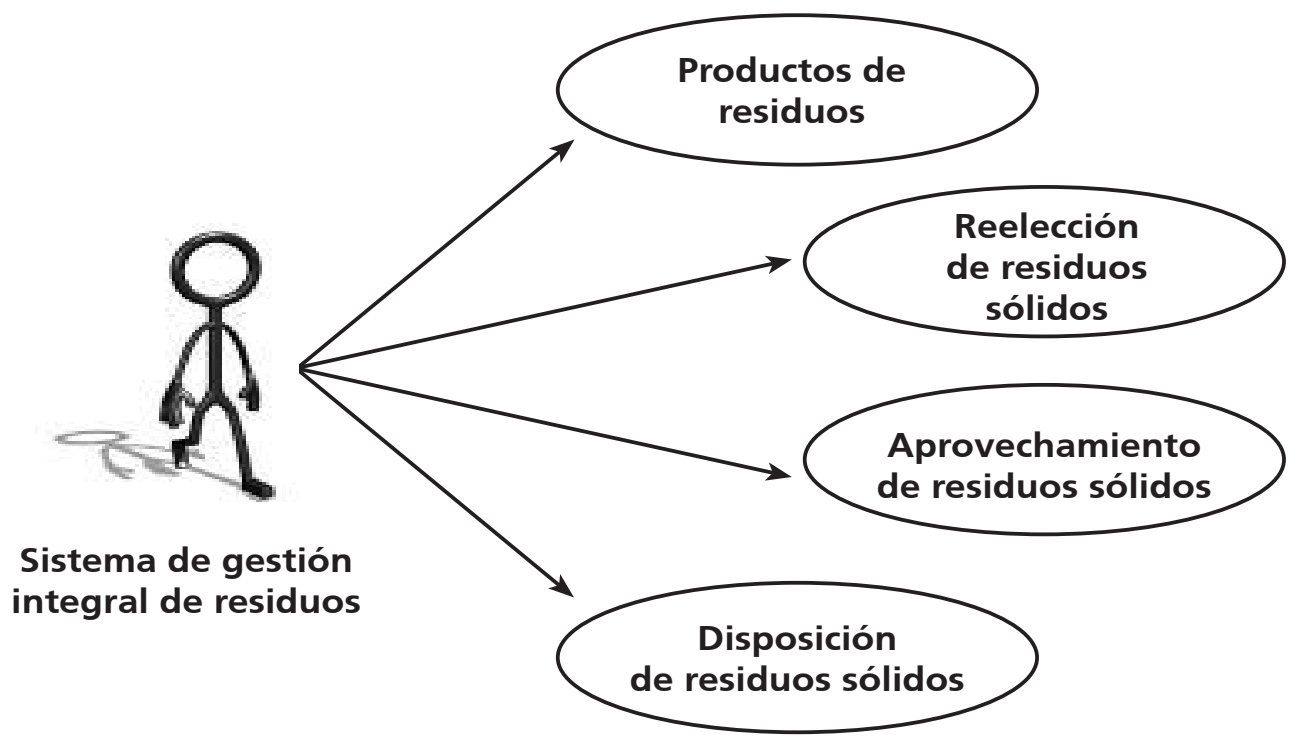

Fuente. Elaboración propia de los autores, 2014.

Es así como se muestra el panorama global de acciones a las que se tendrá que enfrentar el sistema de gestión integral de los residuos: producción, recolección, aprovechamiento y disposición final de residuos especiales. 
Figura 3. Diagrama de clases de la estructura global de fases tecnológicas del CIRRS.

\begin{tabular}{|l|}
\hline Producción de residuos sólidos \\
\hline - Volúmenes: int \\
- Tipos de residuos: int \\
- Fuentes de generación de \\
residuos: int \\
- Fuentes de generación de \\
residuos: int \\
- Localización de fuentes de \\
generación de residuos: int \\
\hline + Reducción en la fuente ( ): void \\
+ Preselección ( ): void \\
+ Cambio de hábitos de consumo \\
(): void
\end{tabular}

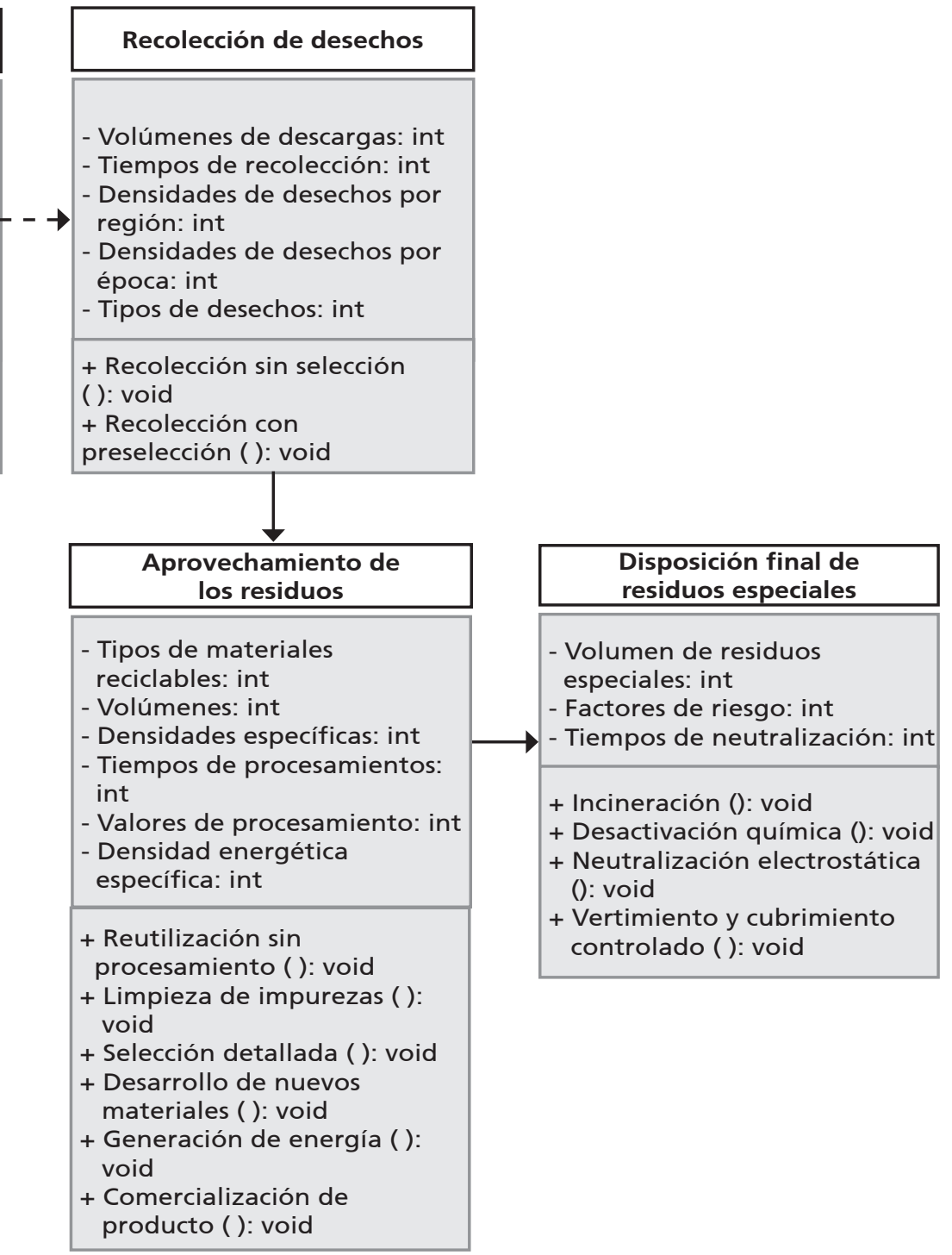

Fuente. Elaboración propia de los autores, 2014.

De acuerdo con lo anterior (Figura3), se ilustran de forma más detallada las fases del proceso tecnológico de la Gestión de los Residuos especificando las variables de cada etapa y los subprocesos que se deberán implementar para llevar a cabo cada fase; por ejemplo, en la fase de aprovechamiento se muestran p.210 como variables los volúmenes y tipos
materiales, sus densidades específicas, los tiempos y valores estimados de procesamiento, entre otros; por otro lado, se complementa con los subprocesos de la fase de aprovechamiento que se indican en la casilla inferior de la clase limpieza de impurezas, selección detallada de materiales, generación de energía y demás. Por último, el diagrama de secuencias ilustra la forma como se comportarán las fases de gestión entre sí, a través del intercambio de mensajes de una etapa a la otra. Es decir, la etapa de recolección envía un mensaje a la etapa de Aprovechamiento de Residuos donde se encontrará el CIRRS, sobre los tipos de materiales colectados y sus densidades específicas para que así se puedan hacer los ajustes de los subprocesos para la automatización total de la etapa en procura de obtener altos niveles de eficiencia en la producción del CIRRS. 
Figura 4. Diagrama de secuencias del proceso tecnológico global del CIRRS.

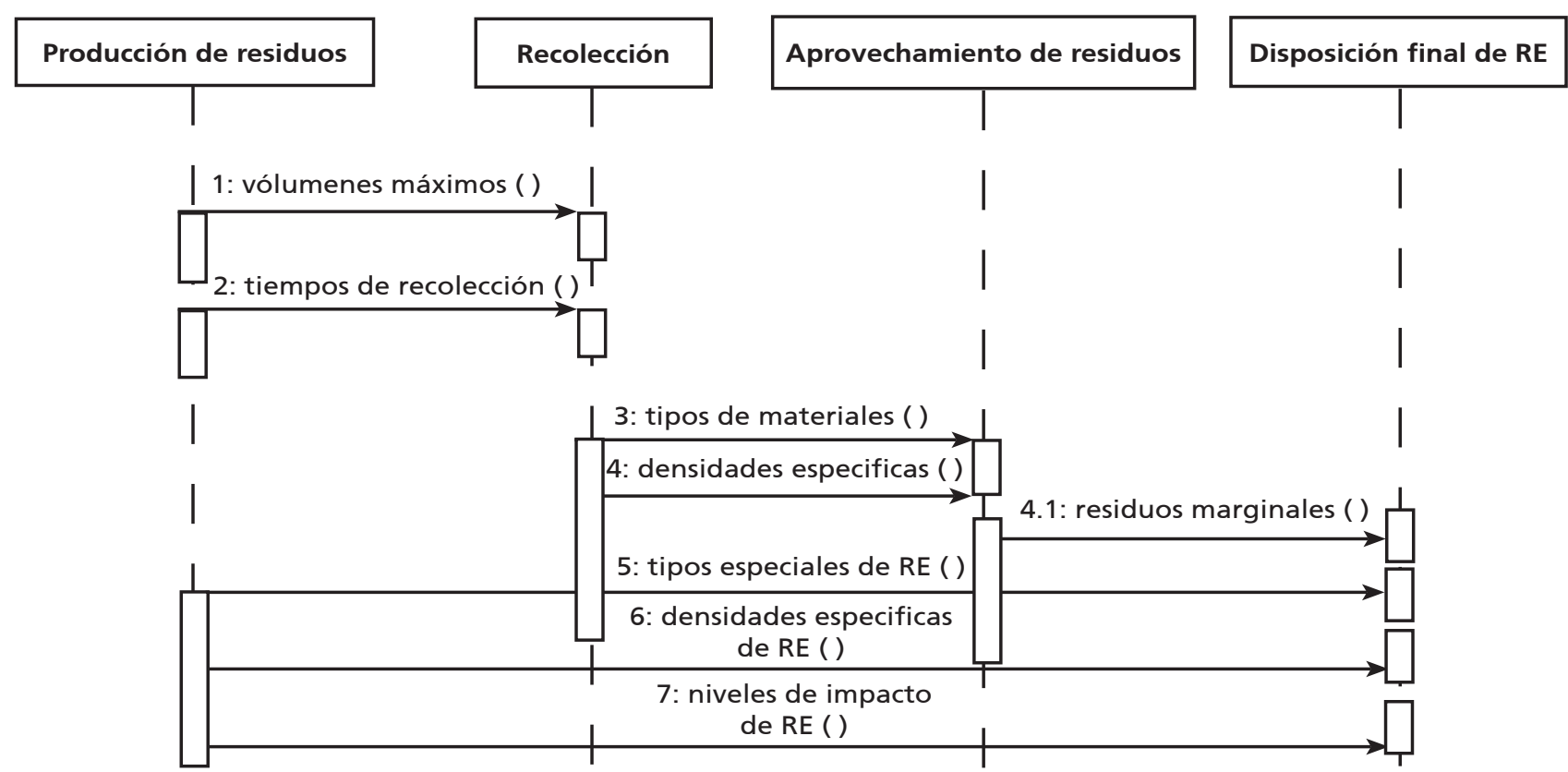

Fuente. Elaboración propia de los autores, 2014.

UML es utilizado de manera universal en la modelación de procesos y de sistemas automatizados, los cuales son el soporte tecnológico para el manejo integral de residuos. La principal cualidad del UML, es la independencia de cualquier tecnología o lenguaje de programación y un carácter holístico (Pérez et al., 2005); es así como se escoge UML como sistema de desarrollo de Software libre y de soluciones de sistemas automatizados y de gestión en diversos ámbitos del diseño de ingeniería.

\subsection{Proceso de reciclaje en el CIRRS}

El proceso dentro del CIRRS, comprende una serie de etapas desde el acopio inicial de los residuos en las tolvas de recepción, pasando por las etapas de descompactación, fraccionamiento, desaireación y desactivación de los residuos; en seguida, se pasa a las fases electrostáticas y electromagnéticas de selección, para continuar con fases de selección manual y de procesamiento diferenciado de los materiales a reciclar.

\subsubsection{Acopio inicial}

Una vez realizada la recolección de desechos, estos son llevados a tanques de acopio de volumen variable, dependiendo del estudio sobre la cantidad de basuras que se generan diariamente en una región. En estos tanques, con ayuda de movimientos oscilatorios, aire a alta presión llevado por compresores y un aumento paulatino de hasta los 40 - 50 grados centígrados, se reduce la humedad de los desechos, además de que se lleva a cabo un proceso primario de selección de basura eliminando desechos de $<10 \mathrm{~mm}$ de espesor que serán procesados en ciclos aparte, mediante la utilización de un conjunto de diferentes rejillas, colocadas en la parte inferior de los tanques. A continuación, se muestra la zona de acopio inicial con área de descargue para vehículos de carga (Ver figura 5). Las zonas de acceso vial, deben concertarse dentro del marco legal del Plan de Ordenamiento Territorial (POT) de los municipios (Ministerio del Interior y de Justicia de Colombia, Ley orgánica de ordenamiento territorial, 2011). De la zona de descargue, se pasa a las bandejas de recibo donde se vierten y se descompactan de manera preliminar los residuos; aquí se lleva a cabo el rompimiento de bolsas y de algunas estructuras de desechos sobredimensionadas para poder ser acomodadas en las canastillas de los 
canjilones. Esta fase se ha diseñado para ser realizada de manera manual, primero, por la diversidad de tareas de descompactación, debido a lo heterogéneo del material a procesar; y segundo, por el compromiso social de inclusión de las comunidades cercanas al CIRRS.

Figura 5. Vista de la zona correspondiente a la etapa 1 del proceso de reciclaje.

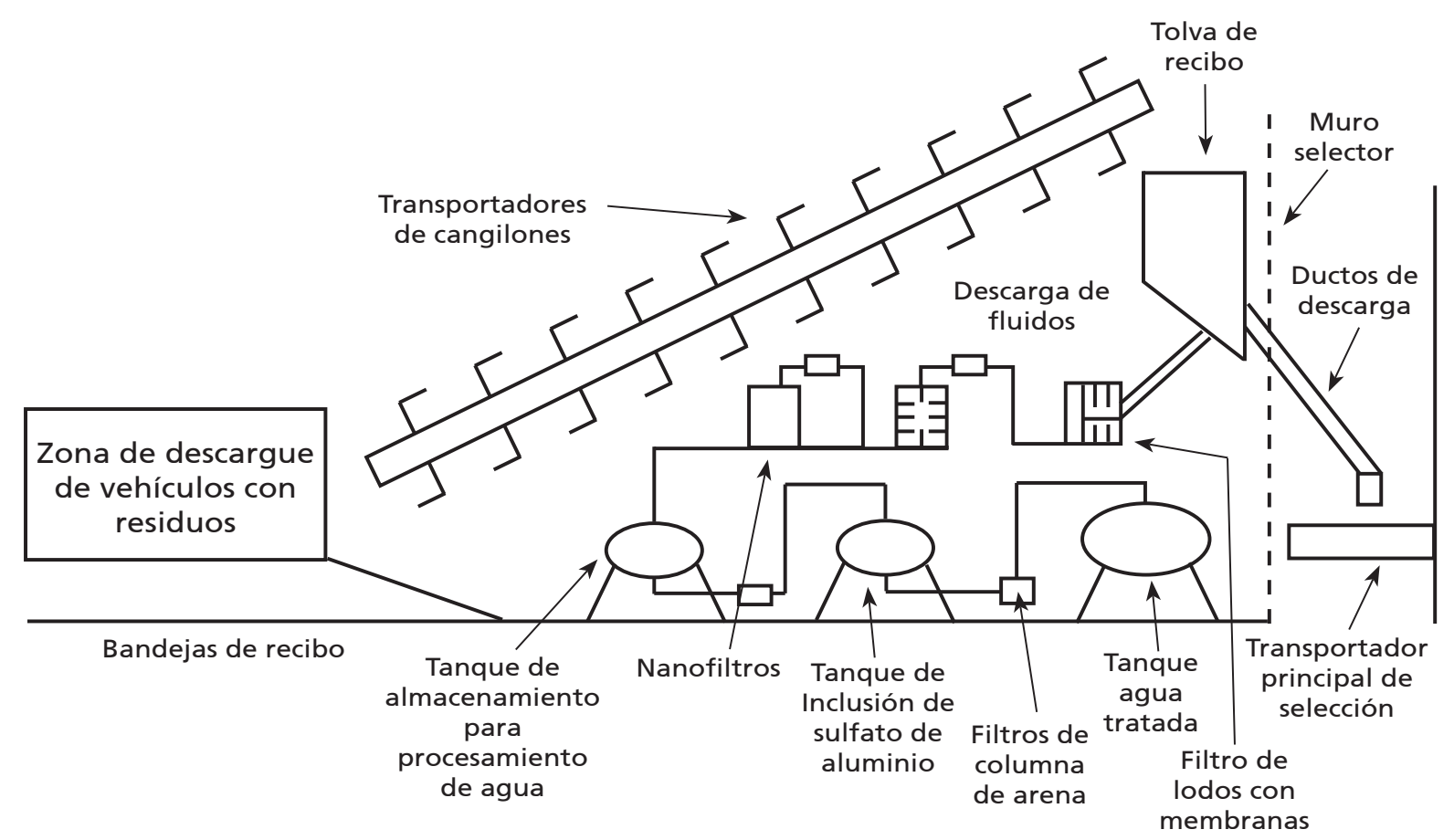

Fuente. Elaboración propia de los autores, 2014.

Con ayuda de los transportadores de canjilones se conducen los residuos a las tolvas, donde se cuenta con elementos de corte para una segunda fase de descompactación y fraccionamiento del material a procesar. En la tolva, se da la alternancia de ciclos de corte, rotación de cuchillas, traslación del sistema de corte y aireación continua con ayuda de compresores y de inclusión de fases de calentamiento entre 40 y 50 grados centígrados. Debido a la posibilidad de encontrar residuos con altos potenciales reactivos (sustancias con niveles de acidez altos $\mathrm{PH}>7.5$ ), se ha diseñado el sistema de tolva con un dispensador-dosificador de cal, como elemento base para la neutralización de elementos con niveles de acidez que puedan afectar a los operarios y/o a partes estructurales del sistema (Barraud et al., 2009). La parte final del sistema de tolvas tiene dos derivaciones: una para los residuos sólidos fraccionados, que van a una banda transportadora principal y otra, para la conducción de líquidos residuales que se tienen del proceso de reducción de la humedad.

\subsubsection{Procesamiento de líquidos residuales}

Los líquidos residuales derivados en los procesos de descompactación, fraccionamiento y reducción de humedad y desactivación de niveles de $\mathrm{PH}$ altos, se envían inicialmente a filtros de lodos compuestos por membranas micro-texturizadas, intercambiables durante labores de mantenimiento; luego, se envían a filtros de columna de arena granulada. El líquido obtenido hasta este momento es bombeado a una presión relativamente alta (150 PSI), para hacerla pasar por una estructura transversal de nano filtros (Van der Bruggen et al., 2008). Posterior al nano-filtrado, se pasa a un tanque de almacenamiento para el procesamiento del líquido, el cual se bombea a un tanque de inclusión de sulfato de aluminio como coagulante y de allí, se pasa a una fase de adición de cloro dosificado. El complemento en la fase de purificación del agua, se realizará con la implementación de un sistema de purificación a través de colectores y paneles fotovoltaicos. Finalmente, se obtendrá agua tratada que será utilizada para suplir necesidades en el centro integral de reciclaje (Vivar et al., 2012). 


\subsubsection{Fase de selección gruesa}

En esta fase, los desechos son conducidos por una banda transportadora central que sale de las tolvas; aquí los operarios realizan una labor de selección, identificando grupos de desechos y clasificación que se ha establecido de la siguiente forma: material orgánico, metálicos, vidrios, materiales de desechos de construcción, plásticos, maderables, papel, cartón, residuos especiales (hospitalarios y no reciclables en general). Se ilustran las fases de procesamiento y de selección preliminar de los materiales residuales, así como los puntos de selección, donde se tendrá personal de la región cercana al CIRRS (Figura 6). Adicionalmente, se considera que durante el año, el volumen y tipo de residuos cambian en su composición, razón por la cual el sistema del centro contará con la posibilidad de adecuarse a regímenes particulares de trabajo con ahorro de energía y menor desgaste en algunas maquinarías, de ser necesario. Para ello, se plantea trabajar con sistemas de automatización industrial para manufactura flexible apoyado en PLC (controladores lógicos programables) con esquemas de producción adaptativos en función de la evaluación dinámica de los flujos de proceso, tiempos y necesidades ajustadas a la demanda (Huang et al., 2013).

Figura 6. Vista general del sistema de bandas transportadoras para la selección de materiales

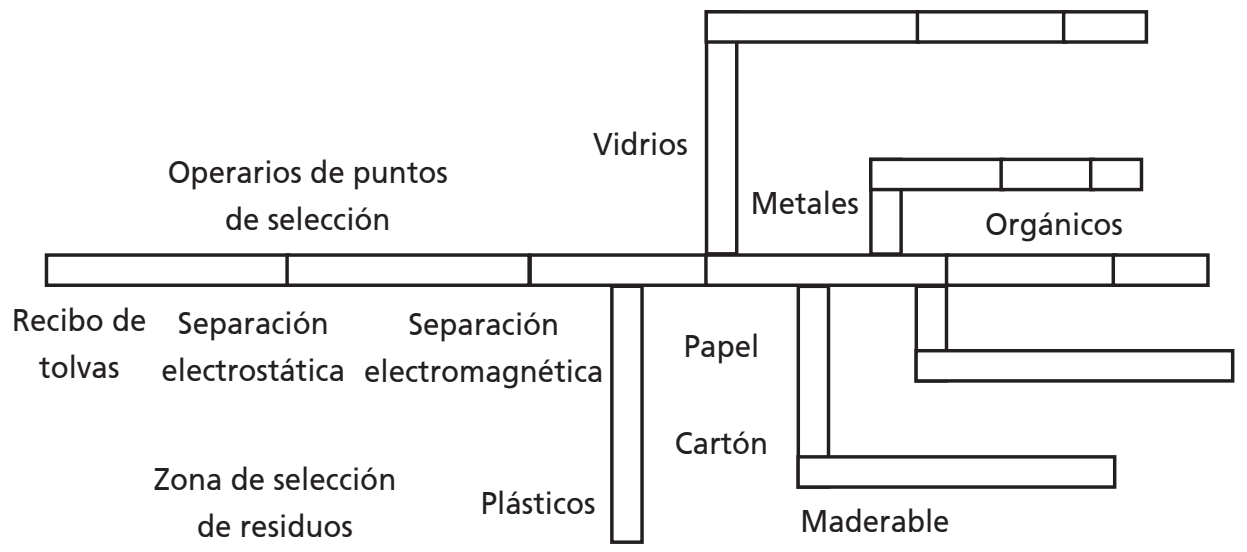

Fuente. Elaboración propia de los autores, 2014.

El trabajo del centro es optimizado gracias a la posibilidad de realizar por ciclos el tratamiento sobre grupos concretos de desechos. Los valores estimados del proyecto, que son producto de la investigación exploratoria inicial, se representan a continuación (Tabla 1). Las dimensiones estimadas del centro son de 110 metros de largo, 30 metros de ancho y diez metros de altura, para una capacidad de procesamiento de 100 toneladas diarias. El número de puestos de trabajo directos para la operación del centro, se estima en 106 (80 operativos y 26 entre administrativos y servicios generales); los costos de operación del proyecto, hacen parte de una investigación exploratoria inicial (Tabla 2). Los estimativos de ventas de material reciclado para el centro, producto de modelación matemática de las cantidades y porcentajes de desechos que se esperan aprovechar, se muestran más adelante (Tabla 3). Los valores estimados de ventas, se reflejan en dos escenarios: uno, realizando el reciclaje simple de los materiales mediante su selección, limpieza y posterior embalaje para ser reutilizados en forma natural. En el segundo escenario, con procesamiento se llevan a cabo etapas adicionales como el peletizado y aglutinado de plásticos, la fabricación de aglomerados con las maderas y papeles, entre otros procesos, lo que conlleva a que el precio de venta sea mayor con relación a productos no procesados. Con respecto de la propiedad y administración del CIRRS, se proponen escenarios de administración de tipo estatal, privada o mixta. En función al tipo seleccionado, se indicará el margen de utilidades respectivo para los ejecutores y administradores del proyecto. Por el momento, este aspecto no se aborda a profundidad en este artículo. 
Tabla 1. Estimativos de inversión inicial para el proyecto en pesos Colombianos (COP)

\begin{tabular}{|l|r|}
\hline Sistema contenedores (2) & $102^{\prime} 500.000$ \\
\hline Sistema de Bandas & $37^{\prime} 050.000$ \\
\hline Embalaje simple & $347^{\prime} 000.000$ \\
\hline Procesamiento Plásticos & $391^{\prime} 500.000$ \\
\hline Biodigestores & $372^{\prime} 000.000$ \\
\hline Procesamiento maderables & $162^{\prime} 200.000$ \\
\hline Procesamiento vidrio & $32^{\prime} 200.000$ \\
\hline Central termoelétrica & $918^{\prime} 000.000$ \\
\hline Páneles solares & $110^{\prime} 000.000$ \\
\hline Metales & 54.400 .000 \\
\hline Obra civil & $850^{\prime} 000.000$ \\
\hline Infraestructura de oficinas & $158^{\prime} 300.000$ \\
\hline Y administración generales & 110.414 .000 \\
\hline Adecuación de micro relleno & $450^{\prime} 000.000$ \\
\hline Total & 4.095 .564 .000 \\
\hline
\end{tabular}

Fuente. Elaboración propia de los autores, 2014.

Tabla 2. Gastos total servicios y mantenimiento al mes Servicios mensuales

\begin{tabular}{|l|r|}
\hline Agua & $1.500 .000,00$ \\
\hline Teléfono e Internet & $1.000 .000,00$ \\
\hline Energía eléctrica & $18.000 .000,00$ \\
\hline Impuestos varios & $6.500 .000,00$ \\
\hline Predial diferido & $12.000 .000,00$ \\
\hline
\end{tabular}

Nómina mensual en planta proyectada por secciones

\begin{tabular}{|l|r|}
\hline Contenedor & $13.650 .000,00$ \\
\hline Sistema de bandas & $16.160 .000,00$ \\
\hline Embalaje & $12.390 .000,00$ \\
\hline Plásticos & $13.760 .000,00$ \\
\hline Biogás & $11.046 .000,00$ \\
\hline Maderables & $13.626 .000,00$ \\
\hline Vidrio & $6.523 .000,00$ \\
\hline Metal & $12.546 .000,00$ \\
\hline Materiales de construcción & $12.750 .000,00$ \\
\hline ADMINISTRATIVOS (NÓMINA) & $5.000 .000,00$ \\
\hline Administrativos nomina) & $119.400 .000,00$ \\
\hline Otros & $850.000,00$ \\
\hline TOTAL & $276.701 .000,00$ \\
\hline
\end{tabular}

Fuente. Elaboración propia de los autores, 2014.
Tabla 3. Estimativos de ventas mensual de productos del CIRRS

\begin{tabular}{|l|r|r|}
\hline \multicolumn{1}{|c|}{ Material } & $\begin{array}{c}\text { Ventas de } \\
\text { producto } \\
\text { Sin procesar } \\
\text { (Toneladas } \\
\text { aprovechadas } \\
\text { al mes* precio- } \\
\text { promedio) }\end{array}$ & $\begin{array}{c}\text { Ventas de } \\
\text { producto } \\
\text { procesado } \\
\text { (Toneladas } \\
\text { aprovechadas } \\
\text { al mes* precio- } \\
\text { promedio) }\end{array}$ \\
\hline Papel Blanco & $21^{\prime} 375.000$ & $35^{\prime} 625.000$ \\
\hline Periódico & $6^{\prime} 480.000$ & $8^{\prime} 100.000$ \\
\hline Plegado y otros & $10^{\prime} 200.000$ & $15^{\prime} 300.000$ \\
\hline Metal & $26^{\prime} 100.000$ & $39^{\prime} 150.000$ \\
\hline Cascajo & $5^{\prime} 700.000$ & $12^{\prime} 540.000$ \\
\hline Plásticos & $480^{\prime} 00.000$ & $750^{\prime} 000.000$ \\
\hline Vidrio & $1^{\prime} 620.000$ & $3^{\prime} 240.000$ \\
\hline Cartón & $24^{\prime} 300.000$ & $28^{\prime} 350.000$ \\
\hline TOTALES & $575 .^{\prime} 775.000$ & $893^{\prime} 305.000$ \\
\hline
\end{tabular}

Fuente. Elaboración propia de los autores, 2014

\subsubsection{Limpieza electrostática y electromagnética}

Mediante la utilización de cepillos electrostáticos y electroimanes, se libra a los diferentes tipos de desechos, en especial a los orgánicos, de impurezas metálicas que dificultan en gran medida el posterior procesamiento de los mismos. La técnica a implementar, será la de la creación de coronas de carga para separar mezclas no metálicas de mezclas metálicas y que resulta aplicable para la separación de materiales conductores y dieléctricos (Gente et al., 2003; Hou et al., 2010; Li et al., 2007). En cuanto al proceso de selección de los plásticos, se adoptó el proceso de separación tribo-electrostática que utiliza la diferencia de propiedades de la superficie de los diferentes materiales que están cargados de manera opuesta, que deflactan el campo eléctrico y se colectan separadamente. El método tribo-electrostático, ofrece importantes ventajas como la alta eficiencia, el bajo costo y sus bajos niveles contaminantes (Guiging et al., 2012). Adicionalmente, se ha dispuesto implementar una fase electrostática de desactivación de iones para eliminación de algunos radicales, en particular sales amoniacales, mercurio, yodo y otros. 


\subsubsection{Selección de subtipos de materiales}

El propósito general es preparar los materiales de desecho para su procesamiento final, teniendo en cuenta que dentro de las diversas clases de materiales existen subdivisiones: colores, texturas, tamaños y otros que al momento de su reutilización son factores importantes para tener en cuenta.

\subsubsection{Almacenamiento y embalaje}

Considerando que todos los desechos no serán procesados de una sola vez, ya sea porque la cantidad sea insuficiente en el momento dado o porque así lo dicte la política de mercadeo (productos con mayor demanda que otros), se sugiere su adecuado almacenamiento. Se dispondrá de un sistema de prensado, embalaje y posterior almacenamiento mientras se esperan las fases posteriores de procesamiento.

\section{PROCESAMIENTO DE MATERIALES}

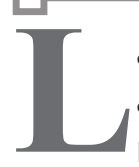
a idea es la producción de nuevos materiales a partir de los desechos que se tengan en el momento y de ser posible, la reutilización directa de muchos de ellos que ya se encuentren en buena forma después de las fases anteriormente mencionadas. La comercialización de los nuevos productos e insumos, permitirá en gran medida la financiación de los costos totales de funcionamiento de la planta una vez esté instalada.

En el caso de la materia orgánica, se proyecta utilizar un $50 \%$ del volumen aprovechable para el proceso de obtención de abonos a partir de compostaje, lombricultura y cultivo de microorganismos, permitiendo la orientación a tipos específicos de abonos. El volumen restante, de tipo animal particularmente, será destinado a la obtención de biogás mediante la implementación de biodigestores automatizados (Arias et al., 2012). Esto permite un impacto medioambiental favorable y un ahorro energético considerable si se compara con las inversiones en opciones convencionales de abastecimiento de gas (Ramírez et al.,1996). La producción de gas estaría orientada inicialmente al abastecimiento de motores, quemadores y de la Pequeña Central Termoeléctrica (PCT) que se instalaría en el CIRRS; los excedentes se dedicarían a la comercialización. En cuanto al papel, el cartón y sus derivados, se plantean fases de procesamiento que incluyen corte y triturado previo, para luego hacerlos reaccionar con agentes reactivos que los conviertan en la materia prima original, a un bajo costo. Los compuestos reactivos, como hidróxido de potasio, pueden ser extraídos de las aguas residuales que trata el centro, logrando así desde el punto de vista técnico, cadenas retroalimentadas con altos niveles de optimización, aspecto muy importante en la evaluación de cualquier proyecto tecnológico. Alrededor de un $25 \%$ del papel, cartón y desechos maderables, se destinarán a la fabricación de materiales alternativos tipo madeflex, que incluyen en su composición plás-

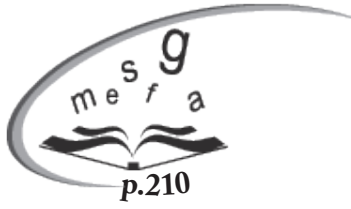
ticos peletizados lo que permitirá el desarrollo de una pequeña microempresa destinada a la fabricación y comercialización de este material, no solo en bruto sino también en terminados, como muebles estanterías y otros afines. Finalmente, una parte del volumen total del papel, cartón y de otros desechos maderables, se destinarán a la incineración para su posterior trituración y pulverización para la producción de carbón. El carbón se destina a comercialización directa, insumo para obtención de abonos y como combustible para la PCT.

Los plásticos, debido a su escaso nivel de biodegradabilidad se convierten en un impermeabilizante de las capas vegetales, produciendo un gran daño a los ecosistemas existentes en la región. Su procesamiento se inicia con el peletizado o corte, luego con su fundición controlada o aglutinado para ser utilizado, ya sea en su forma natural preconcebida tipo PELET aglomerado, aglutinado, o como material de enlace para la fabricación de tintas como tóner de impresoras, así como de materiales constructivos. El uso de residuos de materiales de construcción o cascajo, resulta una alternativa de alto impacto, especialmente para el sector de la construcción mediante la fabricación de aglomerados, con un enfoque de sostenibilidad a pequeñas empresas y reduciendo el impacto ambiental (Damla et al., 2011). 


\section{CLUSTER ECONÓMICO}

$\mathbf{U}$

n Cluster es definido típicamente como una agrupación de empresas localizadas de manera próxima, que se encuentran interconectadas entre sí a través de un campo particular y ligadas por externalidades de varios tipos (Porter, 2003).

A través del CIRRS, se propone la generación de nueva empresa, empleo y el fortalecimiento del tejido social de las comunidades directamente afectadas. La idea del proyecto, se orienta a la integración en los aspectos políticos, sociales económicos y medioambientales que asegure su autosostenibilidad y lo convierta en motor de desarrollo para las regiones. En todas las fases de procesamiento de materiales, se propone la generación de pequeñas empresas dependientes del CIRRS que permitan llevar a cabo una labor de reutilización y aprovechamiento óptimo de los recursos, convirtiéndose en alternativas de empleo directo para los habitantes de la región. El eslabón integrador del Cluster, lo constituirá el CIRRS como tal, siendo proveedor de los insumos para las empresas derivadas y de otro lado, la integración de tipo horizontal.

Por ejemplo, se propone la creación de los siguientes proyectos empresariales, dedicados a la venta de insumos, servicios y productos procesados (Figura 7).

Figura 7. Venta de productos

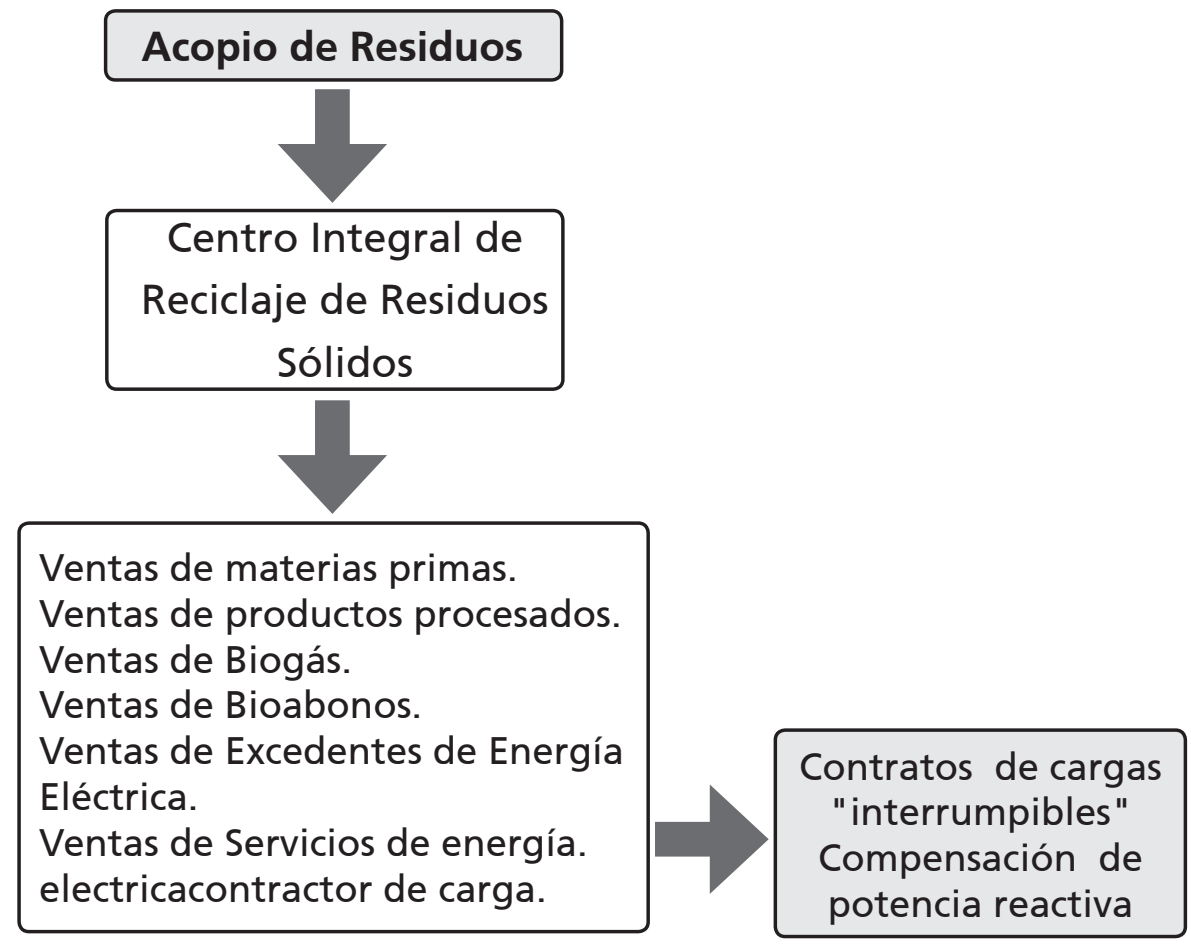

Fuente. Elaboración propia de los autores, 2014. 


\section{MICRO-RED EN GENERACIÓN ELÉCTRICA}

E $n$ el CIRRS, se propone instalar fuentes alternativas de energía que puedan ser utilizadas como fuentes de abastecimiento autónomo del sistema en general y que se proyecten a su vez, como una fuente de Generación Distribuida (GD) incrustada dentro de un Cluster de generación energética de las redes de distribución de energía convencional con que se abastece a la región cercana (Arias et al., 2013; Lasseter et al., 2007).

El CIRRS con su Pequeña Central Termoeléctrica (PCT) y sus fuentes de alternativas de generación, se integrarían al sistema de redes de distribución de la región, formando una micro-red (MR) de GD, que participaría activamente como elemento autogenerador de energía y cogenerador de energía, gracias a la producción simultanea de energía eléctrica y de energía térmica. De igual forma, el CIRRS puede entrar a ofertar en mercado minorista de energía, servicios de tipo complementario para el distribuidor y comercializador de la red eléctrica tales como: alivio de la demanda en horas pico, compensador de potencia reactiva de la red, operar como carga interrumpible, es decir, poder en un momento dado ser desconectado del abastecimiento de la red eléctrica para que el distribuidor atienda otras necesidades prioritarias y mantenga un nivel de confiabilidad en el suministro óptimo dentro de su gestión. Una MR inteligente, tal y como se proyecta el CIRRS, es un sistema que además de abastecer energéticamente a una pequeña comunidad, permite a los consumidores abastecidos por ella controlar y gestionar el uso individual de energía, participar activamente en las operaciones de la red a través de contratos de suministro y de esta manera, contribuir a la disminución de impactos medioambientales como la contaminación electromagnética y sobrecalentamiento de las redes eléctricas (Ramírez, 2013). Las microredes están localizadas, frecuentemente, cerca a las subestaciones de distribución y abastecen cargas industriales y residenciales que pueden incluir elementos de GD y dispositivos de almacenamiento de energía (Hatziargyriou, et al. 2007). Las microredes prestan servicios auxiliares específicos, como la compensación de potencia reactiva y de armónicos (Tedeshi, 2008; Bolognani, 2013).

\subsection{Energía solar fotovoltaica}

El aprovechamiento de la energía solar, se encuentra en experiencias nacionales e internacionales. Para el planteamiento, se han tomado trabajos prácticos realizados en unidades de investigación de reconocidas universidades del país (Santa et al., 2012). El CIRRS, dispondrá de un sistema de paneles solares fotovoltaicos, desplegados en la parte superior o cubierta del complejo construido.

\subsection{Generación eólica}

Para el abastecimiento energético, se propone la inclusión de sistemas aerogeneradores que permiten, a partir de la velocidad del viento, realizar la obtención de energía eléctrica. Los aerogeneradores permiten también realizar funciones de compensación de potencia reactiva en escenarios híbridos de abastecimiento eléctrico por fuentes convencionales y distribuidas (Arias et al., 2013). Para el análisis de las fuentes alternativas energéticas a implementar en el centro Integral de Reciclaje, se ha seguido un modelo de matriz jerarquizada donde se evalúan aspectos políticos, económicos, medioambientales y tecnológicos (Arash et al., 2012). 


\section{CONCLUSIONES}

E sta propuesta para el diseño e implementación de un CIRRS con inclusión de fuentes alternativas de generación, proporciona una visión holística con una perspectiva de solución multifactorial y multiobjetivo del problema de la Gestión Integral de los residuos sólidos en nuestro país.

La revisión de los aspectos legales, tecnológicos, económicos y financieros, así como la figura de propiedad y administración, y energéticos inmersos dentro de la propuesta de solución, brinda un soporte de factibilidad y sostenibilidad importante para la puesta en marcha de soluciones como la que se propone en el CIRRS. El desarrollo de los sistemas biodigestores, los trabajos de investigación sobre implementación de sistemas fotovoltaicos y eólicos, la modelación de las redes de distribución de energía con inclusión de GD, así como las proyecciones de evaluación financiera del proyecto y las perspectivas de impacto social y económico del proyecto realizados por el grupo de investigadores de la Universidad Autónoma, proporcionan una sólida fundamentación teórico-práctica de la viabilidad técnicoeconómica de la presente propuesta.

La inclusión de la solución del CIRRS como un Cluster económico en la producción de materiales y nuevos insumos, su posterior venta y comercialización y la inclusión como elemento activo en la cadena energética a través de sus procesos de autogeneración y cogeneración y la venta de servicios secundarios a la red de distribución tales como la compensación de picos de demanda, de excesos de potencia reactiva en la red, contratos de interrumpibilidad de cargas, entre otros, suponen una solución innovadora y de grandes perspectivas para el momento presente, sobre todo si se tiene en cuenta el nuevo escenario normativo que se ha abierto con la aprobación de la Ley 1715 del 13 de mayo de 2014: Ley de fomento a las fuentes No convencionales de energía (Ley 1715 de 13 de mayo de 2014, por medio de la cual se regula la integración de las energías renovables no convencionales al sistema energético nacional).

Como puede observarse, la idea es que el CIRRS sea una solución sostenible desde el punto de vista energético, al producir su propia energía a partir de la PCT, el sistema de paneles solares, los aerogeneradores y la producción de energía térmica para sus procesos, con ayuda del sistema de biodigestores productores de biogas.

La sostenibilidad económica, se ve sustentada en la diversidad de productos que pueden ofrecerse, sobre todo si se tiene en cuenta la posibilidad de innovar en productos procesados y siendo innovadores con aspectos como la venta de energía y servicios energéticos a la red eléctrica.

Por último, el impacto positivo hacia el medioambiente no solo desde la perspectiva del cuidado de la naturaleza, sino también desde lo social por la creación del empleo, convierten a la propuesta de solución planteada en una solución integral que debe, en la medida de lo posible ser contemplada y muy tenida en cuenta por las autoridades y entidades pertinentes. 


\section{REFERENCIAS BIBLIOGRÁFICAS}

Arias, L., Chica, A., Florez, O y Becerra, M. (2012). UML for the Design of the Biodigester Automation, Congress IEEE- PES, Uruguay.

Arias, L Rivas, E., Garcia, F. (2013). Modelo de gestión de redes de distribución mediante UML y BPL. Revista Tecnura,17(37), pp 109-120.

Astrup, T., Fruergaard, T. y Christensen, T.H (2009).. Recycling of plastic: accounting of greenhouse gases and global warning contributions. Waste Manage. Res. 27, 763-772.

Barraud, J., Creff, Y. y Petit, N. (2009). Ph control of a fed batch reactor with precipitation, Journal of process control, 19(5) 5, pp 888-895.

Bolognani, S. y Zampieri. (2013). A distributed control strategy for reactive power compensation in smart microgrids. Recuperado de: http://arxiv.org/pdf/1106.5626.

Damla, A. y Kasapseckin, M. (2011). Management and recycling of constructional solid waste in Turkey. Procedia Engineering, 21, pp 1072-1077.

Departamento Administrativo Nacional de Estadística (DANE) (2012). Mercado laboral y desempleo. Recuperado de: http://www.dane.gov.co/index.php/mercado-laboral/empleo-y-desempleo

Gente, V., La Marca, F., Lucci, F. y Massacci, P. (2003). Electrical separation of plastics coming from special waste. Waste Manage, 23, 951-958.

Guiging W., Jia, L. y Zhenming, X. (2012). Triboelectrostatic separation for granular plastic waste recycling: A review. School of Environmental Science Engineering, Shangai Jiao Tong University.

Hatziargyriou, N., Asano, H., Travani, R. y Marnay, C. (2007). Microgrids: An Overview of ongoing research, development and demonstration projects IEEE. Power Energy Mag., 5(4), pp. 78-94.

Huang, H., May, M., Wu, C., y Huang, H.M. (2013) Time matrix controller design of flexible manufacturing systems. Computers \& Indsutrial Engineering, 65,(1), pp 28-38.

Lasseter, R. y Piagi, P. (2007). Microgrids and Distributed Generation. Journal of Energy Engineering, American Society of Civil Engineers Microgrid.

Pérez, S., Orejas, F. y Fuentes, N. (2005). Automatización de la arquitectura de componente genéricos usados en UML. (s.l). 
Porter, M. (2003). The Economic Performance of Regions. Regional Studies, 37, pp 549- 578.

Tedeshi, E., Tenti, P. y Mattavelli, P. (2008). Synergistic control and cooperative operation of distribuited harmanonic and reactive compensators. IEEE Power Electron. Specialists Conf., Rhodes, Greece.

Vivar, M., Fuentes, M., Dodd, N., Scott, J., Skryabin, I. y Srithar, K. (2012). First Lab-scala experimental results from a hybrid solar water purification and photovoltaic system. Solar Energy Materials and Solar Cells, 98, pp 260266.

Van Der Bruggen, B., Manttari, M. y Nystrom, M. (2008). Drawbacks of applying nanofiltration and how to avoid them: A review. Separation and Purification Technology, 63(2), pp 251-63.

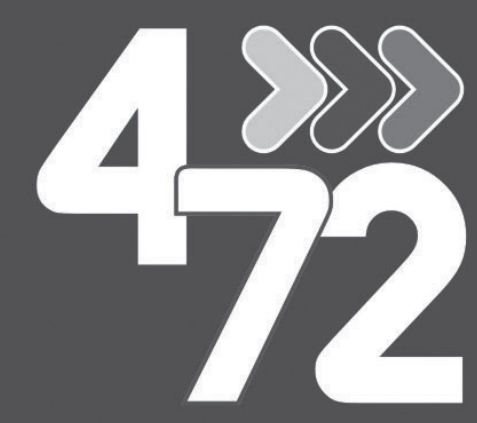

LARED POSTAL DE COLOMBIA

$w w w .4-72 \cdot c 0 m \cdot c 0$

\section{$\checkmark$ Línea de Atención al Cliente Nacional 018000111210}

